\title{
On the Growth of Solutions of a Class of Higher Order Linear Differential Equations with Extremal Coefficients
}

\author{
Jianren Long, ${ }^{1,2}$ Chunhui Qiu, ${ }^{1}$ and Pengcheng $\mathrm{Wu}^{2}$ \\ ${ }^{1}$ School of Mathematical Sciences, Xiamen University, Xiamen 361005, China \\ ${ }^{2}$ School of Mathematics and Computer Science, Guizhou Normal University, Guiyang 550001, China \\ Correspondence should be addressed to Jianren Long; longiianren2004@163.com
}

Received 21 December 2013; Accepted 25 March 2014; Published 10 April 2014

Academic Editor: Mohammad T. Darvishi

Copyright (c) 2014 Jianren Long et al. This is an open access article distributed under the Creative Commons Attribution License, which permits unrestricted use, distribution, and reproduction in any medium, provided the original work is properly cited.

We consider that the linear differential equations $f^{(k)}+A_{k-1}(z) f^{(k-1)}+\cdots+A_{1}(z) f^{\prime}+A_{0}(z) f=0$, where $A_{j}(j=0,1, \ldots, k-1)$, are entire functions. Assume that there exists $l \in\{1,2, \ldots, k-1\}$, such that $A_{l}$ is extremal for Yang's inequality; then we will give some conditions on other coefficients which can guarantee that every solution $f(\not \equiv 0)$ of the equation is of infinite order. More specifically, we estimate the lower bound of hyperorder of $f$ if every solution $f(\neq 0)$ of the equation is of infinite order.

\section{Introduction and Main Results}

We will assume that the reader is familiar with the fundamental results and the standard notations of Nevanlinna theory of meromorphic functions (see $[1,2]$ or [3]). In addition, for a meromorphic function $f$ in the complex plane $\mathbb{C}$, we will use the notations $\rho(f)$ and $\mu(f)$ to denote its order and lower order, respectively.

In order to estimate the rate of growth of meromorphic function of infinite order more precisely, we recall the following definition.

Definition 1 (see [4]). Let $f$ be a meromorphic function in the complex plane $\mathbb{C}$. Then one defines the hyperorder $\rho_{2}(f)$ of $f$ by

$$
\rho_{2}(f)=\limsup _{r \rightarrow \infty} \frac{\log ^{+} \log ^{+} T(r, f)}{\log r} .
$$

Consider the second order linear differential equation

$$
f^{\prime \prime}+A(z) f^{\prime}+B(z) f=0,
$$

where $A$ and $B(\equiv 0)$ are entire functions. It is well known that if $A$ is an entire function, $B(\not \equiv 0)$ is a transcendental entire function, and $f_{1}, f_{2}$ are two linearly independent solutions of (2), then at least one of $f_{1}, f_{2}$ must have infinite order. On the other hand, there are some equations of form (2) that possess a solution $f(\not \equiv 0)$ of finite order; for example, $f(z)=e^{z}$ satisfies $f^{\prime \prime}+e^{-z} f^{\prime}-\left(e^{-z}+1\right) f=0$. Therefore, one may ask, what assumptions on $A(z)$ and $B(z)$ will guarantee that every solution $f \not \equiv 0$ of (2) is of infinite order? From the works of Gundersen (see [5]) and Hellerstein et al. (see [6]), we know that if $A(z)$ and $B(z)$ are entire functions with $\rho(A)<$ $\rho(B)$, or $A(z)$ is a polynomial, and $B(z)$ is transcendental, or $\rho(B)<\rho(A) \leq 1 / 2$, then every solution $f(\not \equiv 0)$ of $(2)$ is of infinite order. More results can be found in [7-12]. For entire solutions of infinite order more precise estimates for their rate of growth would be an important achievement. There are many authors investigating the hyperorder $\rho_{2}(f)$ of solutions of (2), such as Chen and Yang (see $[8,13]$ ) and Kwon (see $[14,15])$.

In this paper, we will introduce the deficient value and Borel direction into the studies of the complex differential equations. In order to give the definition of the Borel direction, we need the following notation. Let $\alpha<\beta$ such that $\beta-\alpha<2 \pi$ and $r>0$; set $\Omega(\alpha, \beta)=\{z: \alpha<\arg z<\beta\}$, $\Omega(\alpha, \beta, r)=\{z: \alpha<\arg z<\beta\} \bigcap\{z:|z|<r\}$.

Definition 2. Let $f$ be a meromorphic function in the complex plane $\mathbb{C}$ with $0<\mu(f)<\infty$. Let $\mu(f) \leq \lambda \leq \rho(f)$ be a finite constant. A ray $\arg z=\theta(0 \leq \theta<2 \pi)$ from the origin 
is called a Borel direction of order $\geq \lambda$ of $f$, if for any positive number $\varepsilon>0$ and for any complex number $a \in \mathbb{C} \bigcup\{\infty\}$, possibly with two exceptions, the following inequality holds:

$$
\limsup _{r \rightarrow \infty} \frac{\log n(\Omega(\theta-\varepsilon, \theta+\varepsilon, r), f=a)}{\log r} \geq \lambda,
$$

where $n(\Omega(\theta-\varepsilon, \theta+\varepsilon, r), f=a)$ denotes the number of zeros, counting the multiplicities, of $f-a$ in the region $\Omega(\theta-\varepsilon, \theta+$ $\varepsilon, r)$.

The fundamental result in angular distribution, due to Valiron, says that a meromorphic function of order $\rho>0$ must have at least one Borel direction of order $\rho$; for example, see [3].

It is well known that deficient values and Borel directions are very important concepts in Nevanlinna theory of meromorphic functions. These two concepts are extensively studied. There is a striking relationship between them which was found by Yang and Zhang and says that, for a meromorphic function $f$ of order $\rho$, the number of deficient values is less or equal to the number of Borel directions of order $\rho$ of $f$. In 1988, Yang extended the above $Y-Z$ inequality to the case of entire function of finite lower order. In order to use Yang's result to study the complex differential equations, we will use the following Theorem which can be easily derived from [16].

Theorem 3 (see [16]). Suppose that $f$ is an entire function of finite lower order $\mu>0$. Let $q(<\infty)$ denote the number of Borel directions of order $\geq \mu$ and $p$ denote the number of finite deficient values of $f$; then $p \leq q / 2$.

Note that Theorem 3 is explicitly stated in [17]. To see the valid of the conclusion of the theorem, we note that, in [17, Corollary 1], Wu has proved that if $f(z)$ is of finite lower order $\mu$ and the number of Borel directions of order $\geq \mu$ is finite, then the order $\rho$ of $f(z)$ is also finite. As each Borel direction of order $\rho$ is also a Borel direction of order $\geq \mu$, this implies that, for $f(z)$, the number of the Borel directions of order $\rho$ is fewer or equal to the number of the Borel directions of order $\geq \mu$. Therefore Theorem 3 follows from Theorem 6.7 in [3].

In the sequel, we will say that an entire function $f$ is extremal for Yang's inequality if $f$ satisfies the assumptions of Theorem 3 with $p=q / 2$.

The simplest entire function extremal for Yang's inequality is $e^{z}$. A little bit complicated example is $A(z)=\int_{0}^{z} e^{-t^{n}} d t$, $(n \geq 2)$. We know that (see [3]) $A(z)$ has $n$ deficient values

$$
a_{l}=e^{i(2 \pi l / n)} \int_{0}^{\infty} e^{-t^{n}} d t, \quad(l=1,2, \ldots, n)
$$

and $q=2 n$ Borel direction $\arg z=((2 k-1) / 2 n) \pi(k=$ $1,2, \ldots, 2 n)$. So $p=q / 2$.

Furthermore, we state the following result due to present authors (see, [18]).

Theorem 4 (see [18]). Let $A$ be an entire function extremal for Yang's inequality, and let $B$ be a transcendental entire function such that $\rho(A) \neq \rho(B)$. Then every solution $f(\not \equiv 0)$ of $(2)$ is of infinite order.
In this paper, we will consider the higher order linear differential equation

$$
f^{(k)}+A_{k-1}(z) f^{(k-1)}+\cdots+A_{1}(z) f^{\prime}+A_{0}(z) f=0,
$$

where $A_{j}(j=0,1, \ldots, k-1)$ are entire functions. Many authors have also investigated the growth of solutions of (5) and obtained lots of results on order and hyperorder of the solutions of (5) (see [19-23]). We will introduce the deficient value and Borel directions into the studies of (5). The main result in the paper is as follows.

Theorem 5. Let $A_{j}(j=0,1, \ldots, k-1)$ be entire functions. Suppose that there exists an integer $l \in\{1,2, \ldots, k-1\}$, such that $A_{l}(z)$ is extremal for Yang's inequality. Suppose that $A_{0}$ is a transcendental entire function with $\rho\left(A_{0}\right) \neq \rho\left(A_{l}\right)$ and $\rho\left(A_{i}\right)<\rho\left(A_{0}\right)$ for $i \neq l(1 \leq i \leq k-1)$. Then every solution $f(\not \equiv 0)$ of (5) satisfies $\rho(f)=\infty$ and $\rho_{2}(f) \geq \rho\left(A_{0}\right)$.

The paper is organized as follows. In Section 2, we will give some lemmas. In Section 3, we will prove Theorem 5. In Section 4, we will discuss some further results related to the two entire coefficients in (5) which are extremal for Yang's inequality.

\section{Lemmas}

In this section, we need some auxiliary results. The following lemma is by Gundersen.

Lemma 6 (see $[24])$. Let $(f, \Gamma)$ denote a pair that consists of a transcendental meromorphic function $f$ and a finite set

$$
\Gamma=\left\{\left(k_{1}, j_{1}\right),\left(k_{2}, j_{2}\right), \ldots,\left(k_{q}, j_{q}\right)\right\}
$$

of distinct pairs of integers that satisfy $k_{i}>j_{i} \geq 0$ for $i=$ $1, \ldots, q$. Let $\alpha>1$ and $\varepsilon>0$ be given real constants. Then the following three statements hold.

(i) There exists a set $E_{1} \subset[0,2 \pi)$ that has linear measure zero, and there exists a constant $c>0$ that depends only on $\alpha$ and $\Gamma$, such that if $\psi_{0} \in[0,2 \pi)-E_{1}$, then there is a constant $R_{0}=R_{0}\left(\psi_{0}\right)>1$ such that, for all $z$ satisfying $\arg z=\psi_{0}$ and $|z|=r \geq R_{0}$ and for all $(k, j) \in \Gamma$, we have

$$
\left|\frac{f^{(k)}(z)}{f^{(j)}(z)}\right| \leq c\left(\frac{T(\alpha r, f)}{r} \log ^{\alpha} r \log T(\alpha r, f)\right)^{k-j} .
$$
by

In particular, if $f$ has finite order $\rho(f)$, then $(7)$ is replaced

$$
\left|\frac{f^{(k)}(z)}{f^{(j)}(z)}\right| \leq|z|^{(k-j)(\rho(f)-1+\varepsilon)}
$$

(ii) There exists a set $E_{2} \subset(1, \infty)$ that has finite logarithmic measure, and there exists a constant $c>0$ that depends only on $\alpha$ and $\Gamma$, such that, for all $z$ satisfying $|z|=r \notin E_{2} \bigcup[0,1]$ and for all $(k, j) \in \Gamma$, inequality (7) holds. holds. 
(iii) There exists a set $E_{3} \subset[0, \infty)$ that has finite linear measure, and there exists a constant $c>0$ that depends only on $\alpha$ and $\Gamma$, such that, for all $z$ satisfying $|z|=r \notin E_{3}$ and for all $(k, j) \in \Gamma$, we have

$$
\left|\frac{f^{(k)}(z)}{f^{(j)}(z)}\right| \leq c\left(T(\alpha r, f) r^{\varepsilon} \log T(\alpha r, f)\right)^{k-j} .
$$
by

In particular, if $f$ has finite order $\rho(f)$, then (9) is replaced

$$
\left|\frac{f^{(k)}(z)}{f^{(j)}(z)}\right| \leq|z|^{(k-j)(\rho(f)+\varepsilon)} .
$$

Let $f$ be an entire function extremal for Yang's inequality $p=q / 2$. Suppose that the rays $\arg z=\theta_{k}(k=1,2, \ldots, q)$ $\left(0 \leq \theta_{1}<\theta_{2}<\cdots<\theta_{q}<\theta_{q+1}=\theta_{1}+2 \pi\right)$ are the $q$ distinct Borel directions of order $\geq \mu$ of $f$. In [17], Wu studied the entire functions which are extremal for Yang's inequality systematically. The following results play an important role in the proof of our results.

Lemma 7 (see [17]). Suppose that $f$ is extremal for Yang's inequality. Then $\mu(f)=\rho(f)$. Moreover, for every deficient value $a_{i}(i=1,2, \ldots, p)$ there exists a corresponding angular domain $\Omega\left(\theta_{k_{i}}, \theta_{k_{i}+1}\right)=\left\{z: \theta_{k_{i}}<\arg z<\theta_{k_{i}+1}\right\}$ such that for every $\varepsilon>0$ inequality

$$
\log \frac{1}{\left|f(z)-a_{i}\right|}>C\left(\theta_{k_{i}}, \theta_{k_{i}+1}, \varepsilon, \delta\left(a_{i}, f\right)\right) T(|z|, f)
$$

holds for $z \in \Omega\left(\theta_{k_{i}}+\varepsilon, \theta_{k_{i}+1}-\varepsilon, r,+\infty\right)=\left\{z: \theta_{k_{i}}+\varepsilon<\arg z<\right.$ $\left.\theta_{k_{i}+1}-\varepsilon\right\} \bigcap\{z: r<|z|<\infty\}$, where $C\left(\theta_{k_{i}}, \theta_{k_{i}+1}, \varepsilon, \delta\left(a_{i}, f\right)\right)$ is a positive constant depending only on $\theta_{k_{i}}, \theta_{k_{i}+1}$, $\varepsilon$ and $\delta\left(a_{i}, f\right)$.

In the sequel, we will say that $f$ decays exponentially to $a_{i}$ in $\Omega\left(\theta_{k_{i}}, \theta_{k_{i}+1}\right)$, if (11) holds in $\Omega\left(\theta_{k_{i}}, \theta_{k_{i}+1}\right)$. Note that if $f$ is extremal for Yang's inequality, then $\mu(f)=\rho(f)$. Thus, for these functions, we need only to consider the Borel direction of order $\rho(f)$.

Lemma 8 (see [18]). Let $f$ be extremal for Yang's inequality. Suppose that there exists $\theta \in \Omega\left(\theta_{j}, \theta_{j+1}\right)(1 \leq j \leq q)$ such that

$$
\limsup _{r \rightarrow \infty} \frac{\log ^{+} \log ^{+}\left|f\left(r e^{i \theta}\right)\right|}{\log r}=\rho(f),
$$

where $\arg z=\theta_{j}(j=1,2, \ldots, q)$ are Borel directions of $f$. Then $\theta_{j+1}-\theta_{j}=\pi / \rho(f)$.

Before stating the following lemmas, for $E \subset[0, \infty)$, we define the Lebesgue measure of $E$ by $\operatorname{mes}(E)$ and the logarithmic measure of $E \subset[1, \infty)$ by $m_{l}(E)=\int_{E}(d t / t)$ and define the upper and lower logarithmic density of $E \subset[1, \infty)$, respectively, by

$$
\begin{aligned}
& \overline{\log \operatorname{den} s} E=\varlimsup_{r \rightarrow \infty} \frac{m_{l}(E \bigcap[1, r])}{\log r}, \\
& \underline{\log \operatorname{den} s} E=\lim _{r \rightarrow \infty} \frac{m_{l}(E \bigcap[1, r])}{\log r} .
\end{aligned}
$$

Lemma 9 (see [25]). Let $f$ be an entire function with $\rho(f)=$ $\rho<1 / 2$ and suppose that $m(r)$ is defined as

$$
m(r)=\inf _{|z|=r} \log |f(z)| .
$$

If $\sigma<\rho$, then the set $\left\{r: m(r)>r^{\sigma}\right\}$ has a positive upper logarithmic density.

Lemma 10 (see [26]). Let $g(r)$ and $h(r)$ be monotone nondecreasing functions on $(0, \infty)$ such that $g(r) \leq h(r)$ for all $r$ outside some set of finite logarithmic measure. Let $\lambda>1$ be a given real constant. Then there exists a constant $r_{0}>0$ such that $g(r) \leq h(\lambda r)$ for all $r \geq r_{0}$.

Lemma 11. Let $f(z)$ be an entire function with order $\rho(0<$ $\rho<\infty)$, let and $\Omega\left(\phi_{1}, \phi_{2}\right)=\left\{z: \phi_{1}<\arg z<\phi_{2}\right\}$ be a sector with $\phi_{2}-\phi_{1}<\pi / \rho$. If there is Borel direction of $f(z)$ in $\Omega\left(\phi_{1}, \phi_{2}\right)$, then there exists at least one of the two rays $L_{j}$ : $\arg z=\phi_{j}(j=1,2)$, without lose of generality, says, $L_{2}$, such that

$$
\limsup _{r \rightarrow \infty} \frac{\log ^{+} \log ^{+}\left|f\left(r e^{i \phi_{2}}\right)\right|}{\log r}=\rho .
$$

Lemma 11 can be founded in [27, Lemma 1], which can be proved by using a result in [28, Page 119-120].

\section{Proof of Theorem 5}

Now we prove our main result.

Since $\rho\left(A_{i}\right)<\rho\left(A_{0}\right)(i \neq l, 1 \leq i \leq k-1)$, we know that for any given constant $\eta$ with $\max \left\{\rho\left(A_{i}\right), i \neq l, 1 \leq i \leq k-1\right\}<$ $\eta<\rho\left(A_{0}\right)$, there exists a constant $R_{1}>0$ such that

$$
\left|A_{i}(z)\right| \leq \exp \left(r^{\eta}\right)
$$

holds for all $|z|=r>R_{1}$.

We consider the following two cases.

Case 1. We suppose that $\rho\left(A_{l}\right)<\rho\left(A_{0}\right)$. Now to the contrary assume that there is a solution $f(\not \equiv 0)$ of $(5)$ with $\rho(f)<+\infty$. We will seek a contradiction. By Lemma 6(ii), there exists a set $E_{1} \subset[1,+\infty)$ that has finite logarithmic measure, such that the following inequality

$$
\left|\frac{f^{(m)}(z)}{f(z)}\right| \leq|z|^{k \rho(f)}, \quad m=1,2, \ldots, k
$$

holds for all $z$ with $|z|=r \notin E_{1} \cup[0,1]$.

We deduce from (17) and (5) that

$$
\begin{aligned}
\left|A_{0}(z)\right| \leq & \left|\frac{f^{(k)}}{f}\right|+\left|A_{k-1}(z)\right|\left|\frac{f^{(k-1)}}{f}\right| \\
& +\cdots+\left|A_{l}(z)\right|\left|\frac{f^{(l)}}{f}\right|+\cdots+\left|A_{1}(z)\right|\left|\frac{f^{\prime}}{f}\right| \\
\leq & |z|^{k \rho(f)}\left(1+\left|A_{k-1}(z)\right|\right. \\
& \left.+\cdots+\left|A_{l}(z)\right|+\cdots+\left|A_{1}(z)\right|\right)
\end{aligned}
$$

holds for all $z$ with $|z|=r \notin E_{1} \bigcup[0,1]$. 
Thus,

$$
T\left(r, A_{0}\right) \leq k \rho(f) \log r+(k-1) T\left(r, A_{i}\right)
$$

holds for all $z$ with $|z|=r \notin E_{1} \bigcup[0,1]$, where $T\left(r, A_{i}\right)=$ $\max _{1 \leq h \leq k-1} T\left(r, A_{h}\right)$. By Lemma 10, we have $\rho\left(A_{0}\right) \leq \rho\left(A_{i}\right)$; this is a contradiction. Therefore, every solution $f(\equiv 0)$ of (5) is of infinite order.

By using similar methods as [14], we can easily prove that $\rho_{2}(f) \geq \rho\left(A_{0}\right)$ in this case. We omit the details here.

Case 2. We suppose that $\rho\left(A_{l}\right)>\rho\left(A_{0}\right)$. Now to the contrary assume that there is a solution $f(\not \equiv)$ of $(5)$ with $\rho(f)<+\infty$. We will seek a contradiction.

Suppose that $a_{i}(i=1,2, \ldots, p)$ are all the finite deficient values of $A_{l}(z)$. Thus we have $2 p$ angular domains $S_{j}=\{z \mid$ $\left.\theta_{j}<\arg z<\theta_{j+1}\right\}(j=1,2, \ldots, 2 p)$. For any $\varepsilon>0$, by using Lemmas 7 and 11, we can easily obtain that $A_{l}(z)$ has the following properties: in each sector $S_{j}$, either there exists some $a_{i}$ such that

$$
\log \frac{1}{\left|A_{l}(z)-a_{i}\right|}>C\left(\theta_{j}, \theta_{j+1}, \varepsilon, \delta\left(a_{i}, A_{l}\right)\right) T\left(|z|, A_{l}\right)
$$

holds for $z \in \Omega\left(\theta_{j}+\varepsilon, \theta_{j+1}-\varepsilon, r,+\infty\right)$, where $C\left(\theta_{j}, \theta_{j+1}, \varepsilon\right.$, $\left.\delta\left(a_{i}, A_{l}\right)\right)$ is a positive constant depending only on $\theta_{j}, \theta_{j+1}, \varepsilon$ and $\delta\left(a_{i}, A_{l}\right)$, or there exists $\theta \in S_{j}$ such that

$$
\limsup _{r \rightarrow \infty} \frac{\log ^{+} \log ^{+}\left|A_{l}\left(r e^{i \theta}\right)\right|}{\log r}=\rho\left(A_{l}\right)
$$

holds. For the sake of simplicity, in the sequel we use $C$ to represent $C\left(\theta_{j}, \theta_{j+1}, \varepsilon, \delta\left(a_{i}, A_{l}\right)\right)$. Note that if there exists some $a_{i}$ such that (20) holds in $S_{j}$, then there exists $\theta$ such that (21) holds in $S_{j-1}$ and $S_{j+1}$. And if there exists $\theta \in S_{j}$ such that (21) holds, then there are $a_{i}\left(a_{i^{\prime}}\right)$ such that (20) holds in $S_{j-1}$ and $S_{j+1}$, respectively.

Without loss of generality, we assume that there is a ray $\arg z=\theta$ in $S_{1}$ such that (21) holds. Therefore, there exists a ray in each sector $S_{3}, S_{5}, \ldots, S_{2 p-1}$, such that (21) holds. By using Lemma 8 , we know that all the sectors have the same magnitude $\pi / \rho\left(A_{l}\right)$.

Firstly, suppose that $\rho\left(A_{0}\right) \geq 1 / 2$. Since $A_{0}(z)$ must have a Borel direction of order $\rho\left(A_{0}\right)$, by using Lemma 11, we can see that there exists a sector $\Omega(\alpha, \beta)(\alpha<\beta)$ such that $\beta-\alpha \geq$ $\pi / \rho\left(A_{0}\right)$ and such that for all the rays $\arg z=\theta(\alpha<\theta<\beta)$ we have

$$
\limsup _{r \rightarrow \infty} \frac{\log ^{+} \log ^{+}\left|A_{0}\left(r e^{i \theta}\right)\right|}{\log r}=\rho\left(A_{0}\right) .
$$

Note that $\rho\left(A_{l}\right)>\rho\left(A_{0}\right)$. It is not hard to see that there exists a sector $\Omega\left(\alpha^{\prime}, \beta^{\prime}\right)\left(\alpha<\alpha^{\prime}<\beta^{\prime}<\beta\right)$ such that there is an $a_{j_{0}}$ such that

$$
\log \frac{1}{\left|A_{l}\left(r e^{i \theta}\right)-a_{j_{0}}\right|}>C T\left(r, A_{l}\right)
$$

holds for all $\alpha^{\prime} \leq \theta \leq \beta^{\prime}$.
By Lemma 6(i), there exist $\theta_{0}\left(\alpha^{\prime} \leq \theta_{0} \leq \beta^{\prime}\right)$ and $R_{2}>0$ such that

$$
\left|\frac{f^{(m)}\left(r e^{i \theta_{0}}\right)}{f\left(r e^{i \theta_{0}}\right)}\right| \leq r^{k \rho(f)}, \quad m=1,2, \ldots, k
$$

holds for all $r>R_{2}$.

Note that

$$
\limsup _{r \rightarrow \infty} \frac{\log ^{+} \log ^{+}\left|A_{0}\left(r e^{i \theta_{0}}\right)\right|}{\log r}=\rho\left(A_{0}\right) .
$$

Thus there is a sequence of $\left\{r_{n}\right\}$ with $\lim _{n \rightarrow \infty} r_{n}=\infty$ such that

$$
\left|A_{0}\left(r_{n} e^{i \theta_{0}}\right)\right| \geq \exp \left(r_{n}^{\rho\left(A_{0}\right)-\varepsilon}\right)
$$

holds for every $0<\varepsilon<\left(\rho\left(A_{0}\right)-\eta\right) / 2$. Therefore, we deduce from (16), (23), and (24) that

$$
\begin{aligned}
\left|A_{0}\left(r_{n} e^{i \theta_{0}}\right)\right| & \\
\leq r_{n}^{k \rho(f)}( & +\left|A_{k-1}\left(r_{n} e^{i \theta_{0}}\right)\right|+\cdots+\left|A_{l}\left(r_{n} e^{i \theta_{0}}\right)-a_{j_{0}}\right| \\
& \left.+\left|a_{j_{0}}\right|+\cdots+\left|A_{1}\left(r_{n} e^{i \theta_{0}}\right)\right|\right) \\
\leq r_{n}^{k \rho(f)}( & +\exp \left(r_{n}^{\eta}\right)+\cdots+\exp \left\{-C T\left(r_{n}, A_{l}\right)\right\} \\
& \left.+\left|a_{j_{0}}\right|+\cdots+\exp \left(r_{n}^{\eta}\right)\right)
\end{aligned}
$$

holds for all sufficiently large $n$. Therefore, combining (26) with (27), we have that

$$
\begin{aligned}
& \exp \left\{r_{n}^{\rho\left(A_{0}\right)-\varepsilon}\right\} \\
& \quad<r_{n}^{k \rho(f)}\left(1+(k-2) \exp \left(r_{n}^{\eta}\right)+\exp \left\{-C T\left(r_{n}, A_{l}\right)\right\}+\left|a_{j_{0}}\right|\right)
\end{aligned}
$$

holds for all sufficiently large $n$. This is a contradiction, so every solution $f(\neq 0)$ of $(5)$ is infinite order in this case.

Secondly, suppose that $0<\rho\left(A_{0}\right)<1 / 2$. By Lemma 9 , there is a sequence of $\left\{r_{n}\right\}$ with $\lim _{n \rightarrow \infty} r_{n}=\infty$ such that for any $\theta \in[0.2 \pi)$, we must have

$$
\left|A_{0}\left(r_{n} e^{i \theta}\right)\right|>\exp \left(r_{n}^{\rho\left(A_{0}\right)-\varepsilon}\right) .
$$

Thus we can get a contradiction by using similar argument for the proof of case $\rho\left(A_{0}\right) \geq 1 / 2$. So every solution $f(\equiv 0)$ of $(5)$ is infinite order in this case.

Lastly, suppose that $\rho\left(A_{0}\right)=0$. Note that $A_{0}(z)$ is a transcendental entire function. By using the results of [29] or [8], for any $\theta \in[0.2 \pi)$, we have

$$
\limsup _{r \rightarrow \infty} \frac{\log \left|A_{0}\left(r e^{i \theta}\right)\right|}{\log r}=\infty .
$$

Thus we can get a contradiction by using similar argument for the proof of case $\rho\left(A_{0}\right) \geq 1 / 2$. Therefore, every solution $f(\equiv 0)$ of (5) is infinite order in this case. 
Next we prove that $\rho_{2}(f) \geq \rho\left(A_{0}\right)$. Firstly, suppose that $\rho\left(A_{0}\right)>0$. By using similar argument as above, there exists $\theta_{0}\left(\alpha^{\prime} \leq \theta_{0} \leq \beta^{\prime}\right)$ and there is a sequence of $\left\{r_{n}\right\}$ with $\lim _{n \rightarrow \infty} r_{n}=\infty$ such that (23) and (26) (or (29)) hold for all sufficiently large $n$.

By Lemma 6(i) that there exist $\theta_{0}\left(\alpha^{\prime} \leq \theta_{0} \leq \beta^{\prime}\right)$ and constants $R_{3}>0, d>0$, such that the following inequality

$$
\left|\frac{f^{(m)}(z)}{f(z)}\right| \leq d T(2 r, f)^{2 k}, \quad m=1, \ldots, k
$$

holds for $|z|=r>R_{3}$.

Hence, calculating at the points $z_{n}=r_{n} e^{i \theta_{0}}$ with $r_{n} \notin$ $\left[0, R_{1}\right] \cup\left[0, R_{3}\right]$, from (16), (23), (26) (or (29)), and (31), we get

$$
\begin{aligned}
\exp \left(r_{n}^{\rho\left(A_{0}\right)-\varepsilon}\right) \leq d T\left(2 r_{n}, f\right)^{2 k} & \left(1+(k-2) \exp \left(r_{n}^{\eta}\right)\right. \\
& \left.+\exp \left\{-C T\left(r_{n}, A_{l}\right)\right\}+\left|a_{j_{0}}\right|\right) .
\end{aligned}
$$

Thus

$$
\varlimsup_{r \rightarrow \infty} \frac{\log ^{+} \log ^{+} T(r, f)}{\log r} \geq \rho\left(A_{0}\right) .
$$

This gives $\rho_{2}(f) \geq \rho\left(A_{0}\right)$.

If $\rho\left(A_{0}\right)=0$, obviously, $\rho_{2}(f) \geq 0$. The proof of Theorem 5 is completed.

\section{Further Results}

In this section, we will study (5) with coefficients $A_{0}$ and $A_{l}$ which are both extremal for Yang's inequality.

Theorem 12. Let $A_{j}(j=0,1, \ldots, k-1)$ be entire functions. Suppose that there exists an integer $l \in\{1,2, \ldots, k-1\}$, such that $A_{l}$ is extremal for Yang's inequality $p_{1}=q_{1} / 2$. Suppose that $A_{0}$ is an entire function extremal for Yang's inequality $p_{2}=$ $q_{2} / 2$ and $\rho\left(A_{i}\right)<\rho\left(A_{0}\right)$ for $i \neq l(1 \leq i \leq k-1)$. Suppose that one of the following conditions holds:

(1) $q_{1} \neq q_{2}$,

(2) $q_{1}=q_{2}$, and the set of Borel directions of $A_{l}$ is different from that of $A_{0}$.

Then every solution $f(\equiv 0)$ of $(5)$ satisfies $\rho(f)=\infty$ and $\rho_{2}(f) \geq \rho\left(A_{0}\right)$.

Proof. We first treat the case that the entire functions $A_{l}$ and $A_{0}$ satisfy condition (1).

Note that if $\rho\left(A_{l}\right) \neq \rho\left(A_{0}\right)$, then the conclusion of Theorem 12 follows from Theorem 5 . Now suppose that $\rho\left(A_{l}\right)=\rho\left(A_{0}\right)=\rho$. We divide the proof into two cases: (a) $q_{1}<q_{2}$ and (b) $q_{1}>q_{2}$.

Now suppose that (a) $q_{1}<q_{2}$ holds. It is easy to see from Lemmas 7 and 8 that there are $q_{2} / 2$ sectors with magnitude $\pi / \rho$ such that

$$
\limsup _{r \rightarrow \infty} \frac{\log ^{+} \log ^{+}\left|A_{0}\left(r e^{i \theta}\right)\right|}{\log r}=\rho,
$$

while there are $q_{1} / 2$ sectors with magnitude $\pi / \rho$ such that

$$
\limsup _{r \rightarrow \infty} \frac{\log ^{+} \log ^{+}\left|A_{l}\left(r e^{i \theta}\right)\right|}{\log r}=\rho .
$$

Note that $q_{1}<q_{2}$. It is easy to see that there exists a sector $\Omega(\alpha, \beta)(0<\alpha<\beta<2 \pi)$ such that, for every $\theta \in(\alpha, \beta)$, $A_{l}(z)$ must be bounded in $\Omega(\alpha, \beta)$, while $A_{0}(z)$ satisfies (34). So, by using the same argument in the proof of Theorem 5 , we can easily prove the theorem.

We next suppose that (b) $q_{1}>q_{2}$. It is not hard to see that there must exist a sector $\Omega(\alpha, \beta)$ such that $A_{l}(z)$ is bounded in $\Omega(\alpha, \beta)$, while for any $\theta \in \Omega(\alpha, \beta) A_{0}(z)$ satisfies (34). By using similar arguments as we did before, we can prove the theorem under the condition that $A_{l}(z)$ and $A_{0}(z)$ satisfy (1).

We turn to the case that $A_{l}(z)$ and $A_{0}(z)$ satisfy (2). In this case, it is easy to see that there exists a sector such that in it $A_{l}(z)$ is bounded, while $A_{0}(z)$ satisfies (34). By using similar arguments as we did before, we can prove the theorem in this case. We omit the details here. The proof of Theorem 12 is completed.

Finally, in [16], we note that if an entire function $f$ is extremal for Yang's inequality $p=q / 2$, then for any positive integer $m, f^{(m)}$ also has some special properties. In the sectors where, for any $\theta, f$ satisfies

$$
\limsup _{r \rightarrow \infty} \frac{\log ^{+} \log ^{+}\left|f\left(r e^{i \theta}\right)\right|}{\log r}=\rho,
$$

for any $\theta, f^{(m)}$ satisfies

$$
\limsup _{r \rightarrow \infty} \frac{\log ^{+} \log ^{+}\left|f^{(m)}\left(r e^{i \theta}\right)\right|}{\log r}=\rho,
$$

$f$ decays to some deficient values exponentially and $f^{(m)}$ decays to 0 exponentially. Therefore, in the same manner as in the proofs of Theorems 5 and 12, we have the following result.

Theorem 13. Let $A_{j}(j=0,1, \ldots, k-1)$ be entire functions. Suppose that there exists an integer $l \in\{1,2, \ldots, k-1\}$, such that $A_{l}$ is extremal for Yang's inequality $p_{1}=q_{1} / 2$. Suppose that $A_{0}$ is a transcendental entire function with $\rho\left(A_{0}\right) \neq \rho\left(A_{l}\right)$ and $\rho\left(A_{i}\right)<\rho\left(A_{0}\right)$ for $i \neq l(1 \leq i \leq k-1)$. Then every solution $f \not \equiv 0$ of

$$
\begin{aligned}
& f^{(k)}+A_{k-1}(z) f^{(k-1)}+\cdots+A_{l}^{(m)}(z) f^{(l)}+\cdots+A_{0}(z) f \\
& \quad=0
\end{aligned}
$$

satisfies $\rho(f)=\infty$ and $\rho_{2}(f) \geq \rho\left(A_{0}\right)$.

Moreover, suppose that $A_{0}$ is an entire function extremal for Yang's inequality $p_{2}=q_{2} / 2$ and that one of the following assumptions holds:

(1) $q_{1} \neq q_{2}$,

(2) $q_{1}=q_{2}$, and the set of Borel directions of $A_{l}$ is different from that of $A_{0}$. 
Then every solution $f(\equiv 0)$ of

$$
f^{(k)}+A_{k-1} f^{(k-1)}+\cdots+A_{l}^{(m)}(z) f^{(l)}+\cdots+A_{0}^{(n)}(z) f=0
$$

satisfies $\rho(f)=\infty$ and $\rho_{2}(f) \geq \rho\left(A_{0}\right)$, where $m$ and $n$ are two nonnegative integers.

Finally we give an example satisfying the conditions of Theorem 12. Let again $A_{n}(z)=\int_{0}^{z} e^{-t^{n}} d t,(n \geq 2)$. So if we let $A_{l}=A_{n}(z), A_{0}=A_{m}(z)=\int_{0}^{z} e^{-t^{m}} d t$ with $n \neq m$ and all other coefficients $A_{i}$ satisfy $\rho\left(A_{i}\right)<\rho\left(A_{0}\right)$ for $i \neq l(1 \leq i \leq k-1)$, then, by Theorem 12(1), every solution $f(\equiv 0)$ of (5) satisfies $\rho(f)=\infty$ and $\rho_{2}(f) \geq \rho\left(A_{0}\right)$. Furthermore, if we let $A_{0}(z)=$ $A_{n}\left(e^{i \theta} z\right)$ with $\theta \in(0, \pi / 2 n)$, then, by Theorem 12(2), every solution $f(\equiv 0)$ of $(5)$ satisfies $\rho(f)=\infty$ and $\rho_{2}(f) \geq \rho\left(A_{0}\right)$.

\section{Conflict of Interests}

The authors declare that there is no conflict of interests regarding the publication of this paper.

\section{Acknowledgments}

The work is supported by the United Technology Foundation of Science and Technology Department of Guizhou Province and Guizhou Normal University (Grant no. LKS [2012] 12), and the National Natural Science Foundation of China (Grant nos. 11171080 and 11171277).

\section{References}

[1] W. K. Hayman, Meromorphic Functions, Oxford Mathematical Monographs, Clarendon Press, Oxford, UK, 1964.

[2] I. Laine, Nevanlinna Theory and Complex Differential Equations, vol. 15 of de Gruyter Studies in Mathematics, Walter de Gruyter, New York, NY, USA, 1993.

[3] L. Yang, Value Distribution Theory, Springer, Berlin, Germany, 1993.

[4] C.-C. Yang and H.-X. Yi, Uniqueness Theory of Meromorphic Functions, vol. 557, Kluwer Academic Publishers, New York, NY, USA, 2003.

[5] G. G. Gundersen, "Finite order solutions of second order linear differential equations," Transactions of the American Mathematical Society, vol. 305, no. 1, pp. 415-429, 1988.

[6] S. Hellerstein, J. Miles, and J. Rossi, "On the growth of solutions of $f^{\prime \prime}+g f^{\prime}+h f=0$," Transactions of the American Mathematical Society, vol. 324, no. 2, pp. 693-706, 1991.

[7] S. B. Bank and I. Laine, "On the oscillation theory of $f$ " $+A f=0$ where $A$ is entire," Transactions of the American Mathematical Society, vol. 273, no. 1, pp. 351-363, 1982.

[8] Z.-X. Chen and C.-C. Yang, "Some further results on the zeros and growths of entire solutions of second order linear differential equations," Kodai Mathematical Journal, vol. 22, no. 2, pp. 273-285, 1999.

[9] G. G. Gundersen, "On the question of whether $f^{\prime \prime}+e^{-z} f^{\prime}+$ $B(z) f=0$ can admit a solution $f \not \equiv 0$ of finite order," Proceedings of the Royal Society of Edinburgh. Section A. Mathematics, vol. 102, no. 1-2, pp. 9-17, 1986.
[10] S. Hellerstein, J. Miles, and J. Rossi, "On the growth of solutions of certain linear differential equations," Annales Academiae Scientiarum Fennicae. Series A I. Mathematica, vol. 17, no. 2, pp. 343-365, 1992.

[11] K.-H. Kwon and J.-H. Kim, "Maximum modulus, characteristic, deficiency and growth of solutions of second order linear differential equations," Kodai Mathematical Journal, vol. 24, no. 3, pp. 344-351, 2001.

[12] M. Ozawa, "On a solution of $w^{\prime \prime}+e^{-z} w^{\prime}+(a z+b) w=0$," Kodai Mathematical Journal, vol. 3, no. 2, pp. 295-309, 1980.

[13] Z.-X. Chen and C.-C. Yang, "On the zeros and hyper-order of meromorphic solutions of linear differential equations," Annales Academice Scientiarum Fennice. Mathematica, vol. 24, no. 1, pp. 215-224, 1999.

[14] K.-H. Kwon, "On the growth of entire functions satisfying second order linear differential equations," Bulletin of the Korean Mathematical Society, vol. 33, no. 3, pp. 487-496, 1996.

[15] K.-H. Kwon, "Nonexistence of finite order solutions of certain second order linear differential equations," Kodai Mathematical Journal, vol. 19, no. 3, pp. 378-387, 1996.

[16] L. Yang, "Deficient values and angular distribution of entire functions," Transactions of the American Mathematical Society, vol. 308, no. 2, pp. 583-601, 1988.

[17] S. Wu, "Some results on entire functions of finite lower order," Acta Mathematica Sinica. New Series, vol. 10, no. 2, pp. 168-178, 1994.

[18] J. R. Long, P. C. Wu, and Z. Zhang, "On the growth of solutions of second order linear differential equations with extremal coefficients," Acta Mathematica Sinica, vol. 29, no. 2, pp. 365372, 2013.

[19] B. Belaïdi, "Growth and oscillation theory of non-homogeneous complex differential equations with entire coefficients," Communications in Mathematical Analysis, vol. 5, no. 2, pp. 13-25, 2008.

[20] B. Belaïdi and S. Abbas, "On the hyper order of solutions of a class of higher order linear differential equations," Analele stiintifice ale Universitatii Ovidius Constanta, vol. 16, no. 2, pp. 15-30, 2008.

[21] Z. Chen, "On the hyper order of solutions of higher order differential equations," Chinese Annals of Mathematics B, vol. 24, no. 4, pp. 501-508, 2003.

[22] Z. Chen, "On the growth of sulutions of a class of higher order differential equations," Acta Mathematica Scientia, vol. 24, pp. 52-60, 2004.

[23] J. Tu and C.-F. Yi, "On the growth of solutions of a class of higher order linear differential equations with coefficients having the same order," Journal of Mathematical Analysis and Applications, vol. 340, no. 1, pp. 487-497, 2008.

[24] G. G. Gundersen, "Estimates for the logarithmic derivative of a meromorphic function, plus similar estimates," Journal of the London Mathematical Society. Second Series, vol. 37, no. 1, pp. 88-104, 1988.

[25] P. D. Barry, "Some theorems related to the $\pi \rho$ theorem," Proceedings of the London Mathematical Society, vol. 21, pp. 334360, 1970.

[26] S. B. Bank, "A general theorem concerning the growth of solutions of first-order algebraic differential equations," Compositio Mathematica, vol. 25, pp. 61-70, 1972.

[27] L. Yang and G. H. Zhang, "Distribution of Borel directions of entire functions," Acta Mathematica Sinica, vol. 19, no. 3, pp. 157-168, 1976 (Chinese). 
[28] M. Tsuji, Potential Theory in Modern Function Theory, Maruzen, Tokyo, Japan, 1959.

[29] P. D. Barry, "On a theorem of Besicovitch," The Quarterly Journal of Mathematics, vol. 14, pp. 293-302, 1963. 


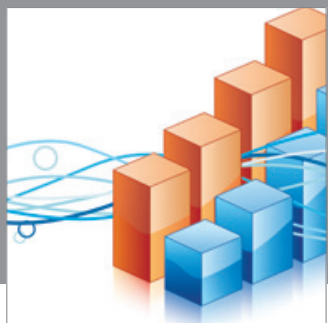

Advances in

Operations Research

mansans

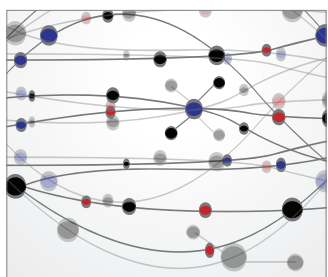

The Scientific World Journal
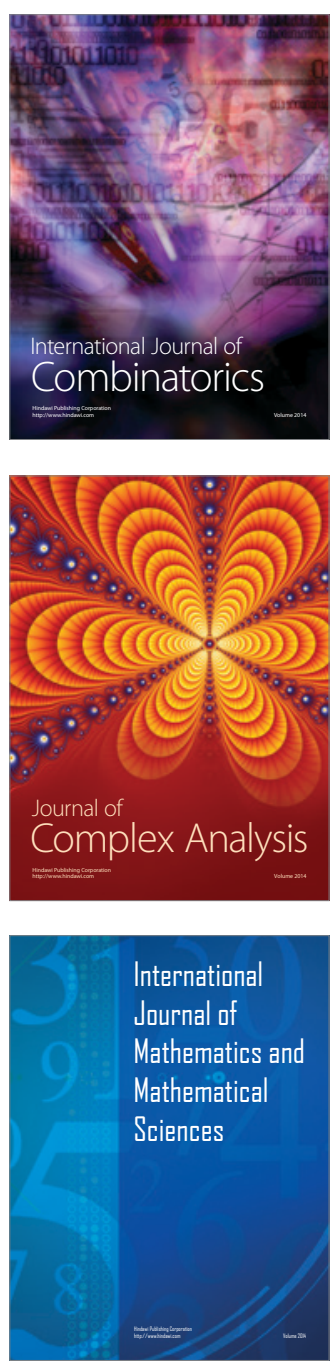
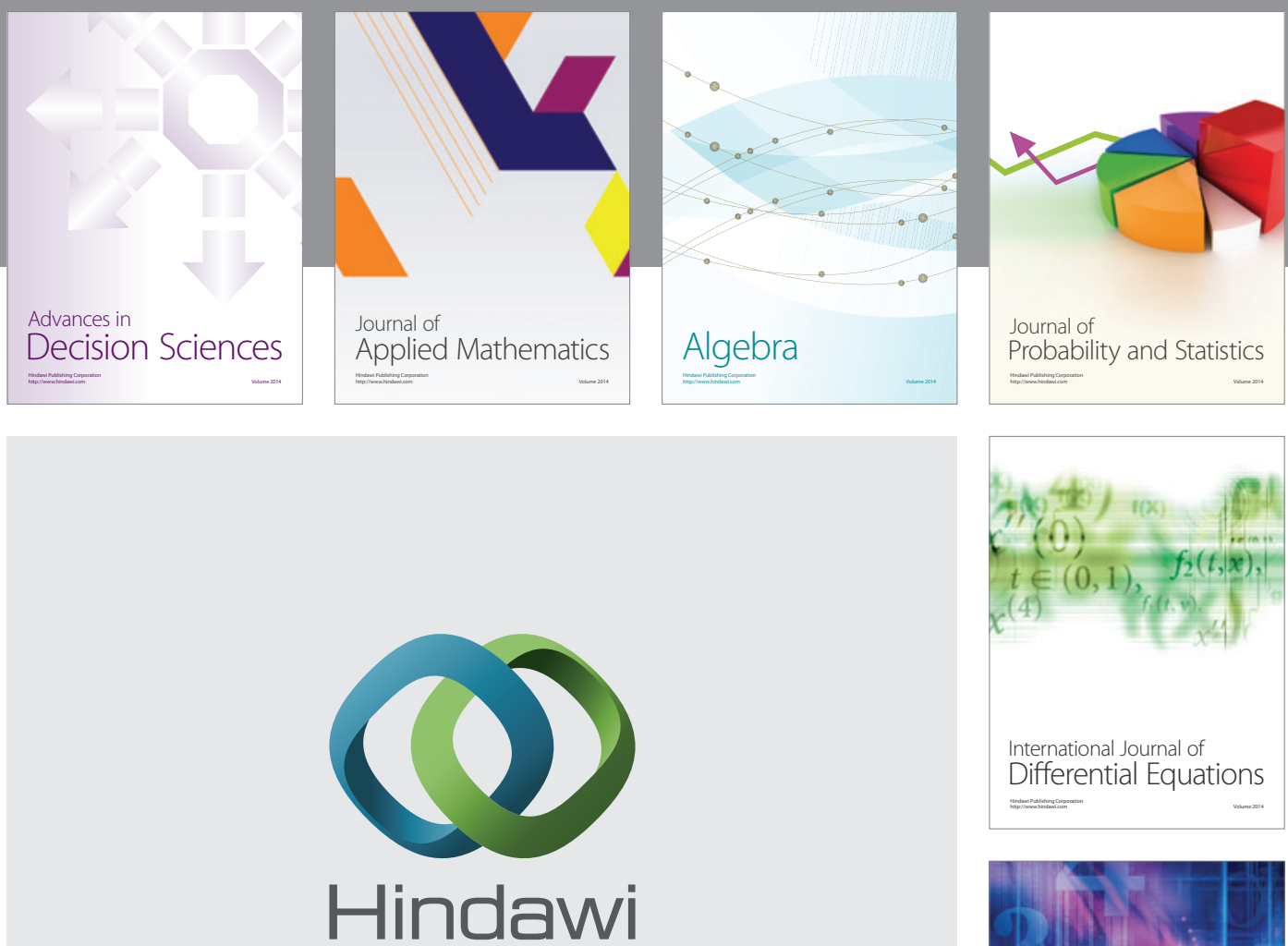

Submit your manuscripts at http://www.hindawi.com
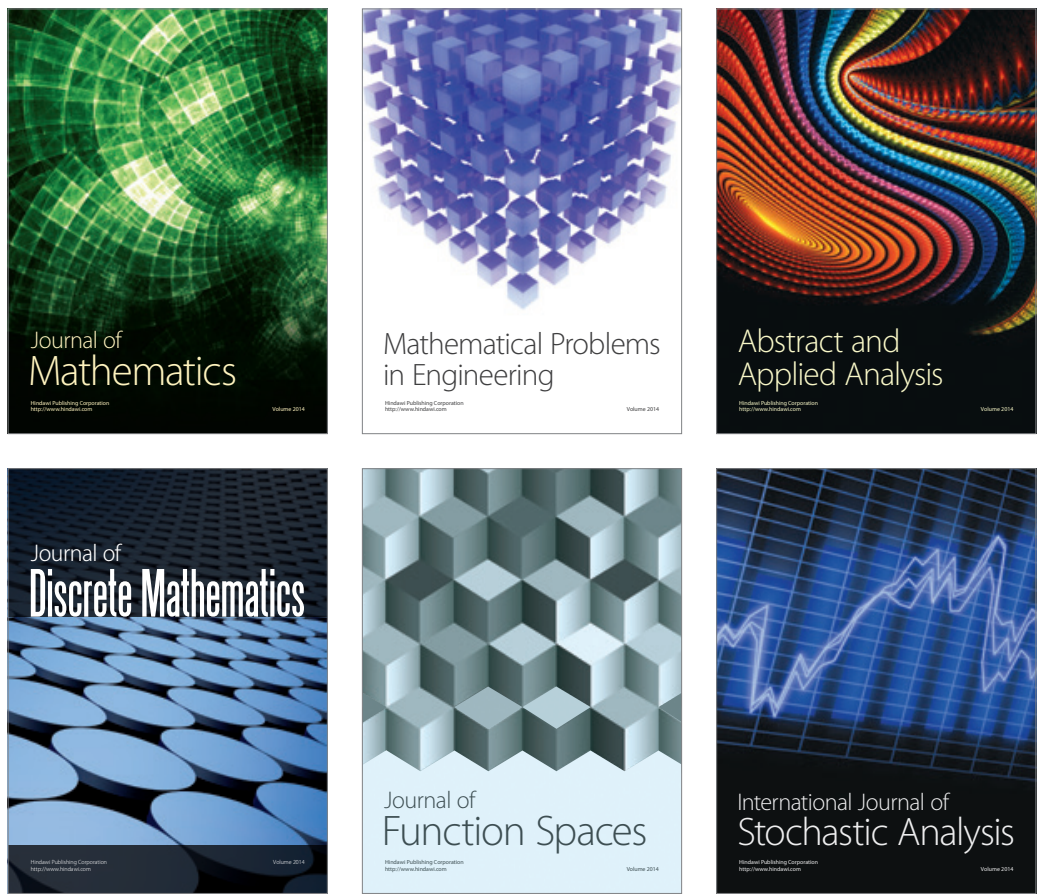

Journal of

Function Spaces

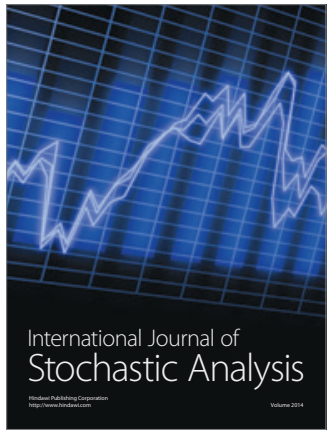

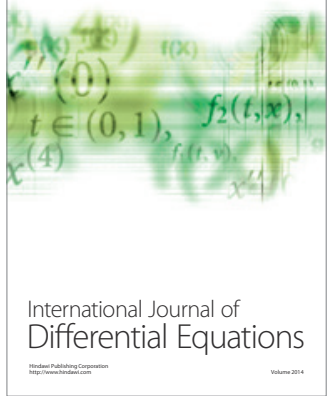
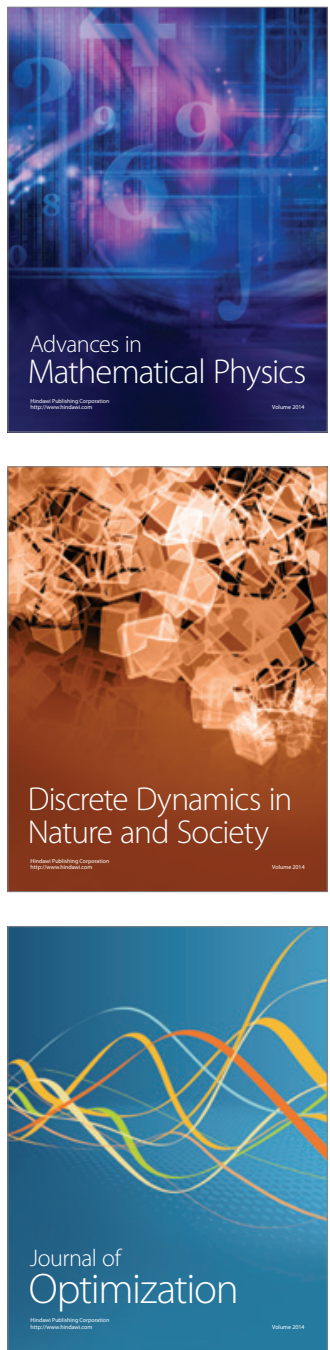